\title{
Indicadores sobre o cuidado a crianças $e$ adolescentes com autismo na rede de CAPSi da região metropolitana do Rio de Janeiro
}

I ${ }^{1}$ Rossano Cabral Lima, 2 Maria Cristina Ventura Couto,

${ }^{3}$ Pedro Gabriel Godinho Delgado, ${ }^{4}$ Bruno Diniz Castro de Oliveira I

Resumo: Os Centros de Atenção Psicossocial Infanto-juvenis (CAPSi) são equipamentos estratégicos para o atendimento de crianças e adolescentes com problemas mentais, incluindo os transtornos do espectro do autismo. Esta pesquisa visou à criação de indicadores sobre o tratamento de autistas nos CAPSi, a partir do discurso de trabalhadores e familiares de 14 CAPSi da região metropolitana do Rio de Janeiro. A metodologia participativa envolveu a realização de 14 grupos focais com trabalhadores de cada CAPSi e três grupos focais com familiares de crianças e adolescentes com autismo atendidos nesses serviços. A transcrição dos registros em áudio dos grupos focais deu origem a cinco "temas ordenadores", reunindo os argumentos produzidos por todos os CAPSi e grupos de familiares. Esse material foi trabalhado numa oficina final, gerando cerca de 130 indicadores, os quais foram agrupados em cinco eixos temáticos: organização do CAPSi; projeto terapêutico individual; atendimento e mobilização dos familiares; rede, território e direitos; formação dos profissionais e processos de trabalho. Tais indicadores podem servir de guias e referências às boas práticas no cuidado com as pessoas com autismo e suas famílias, podendo ser usados para futuras iniciativas de avaliação do atendimento oferecido pelos CAPSi a essa população.

> Palavras-chave: pesquisa avaliativa; autismo; centros de atenção psicossociais infanto-juvenis, saúde mental infanto-juvenil.

\author{
${ }^{1}$ Instituto de Medicina Social, \\ Universidade do Estado do Rio \\ de Janeiro. Rio de Janeiro-RJ, \\ Brasil. Endereço eletrônico: \\ rossanolima@ig.com.br \\ 2 Instituto de Psiquiatria \\ Universidade Federal do Rio \\ de Janeiro. Rio de Janeiro-RJ, \\ Brasil. Endereço eletrônico: cris. \\ ventura13@yahoo.com.br \\ ${ }^{3}$ Instituto de Psiquiatria \\ Universidade Federal do Rio \\ de Janeiro. Rio de Janeiro-RJ, \\ Brasil. Endereço eletrônico: \\ pedrogabrieldelgado.ufrj@ \\ gmail.com \\ ${ }^{4}$ Mestrando, Instituto de \\ Medicina Social, Universidade \\ do Estado do Rio de Janeiro. Rio \\ de Janeiro-RJ, Brasil. Endereço \\ eletrônico: brunodinizcastro@ \\ hotmail.com
}

Recebido em: 17/01/2014 Aprovado em: 26/08/2014 


\section{Introdução}

O autismo é um quadro de etiologia ainda indefinida, que geralmente se instala nos três primeiros anos de vida e é caracterizado pelo comprometimento da interação social e da comunicação e por padrôes restritos e repetitivos de comportamentos, interesses e atividades (BRASIL, 2013a). Estudos epidemiológicos vêm apontando aumento de sua prevalência, mas a interpretação desse dado ainda é alvo de controvérsias (BLAXILL, 2004; COO et al., 2007; FOMBONNE, 2003). Os índices médios para todos os transtornos do espectro autista giram em torno de 6,0/1.000 habitantes (CHARMAN, 2002), mas pesquisas mais recentes apontam taxas de 1,0/100 no Reino Unido (BRUGHA et al., 2011) e 1,0/88 nos EUA (CDC, 2012). No Brasil, estudo piloto realizado em crianças de sete a 12 anos em Atibaia, no estado de São Paulo, encontrou prevalência de 0,3\% (PAULA et al., 2011).

Até o final do século passado, essa população se encontrava majoritariamente fora do campo da saúde mental pública brasileira. Seu cuidado, quando acontecia, era oferecido pela educação, assistência social, por instituiçõos de caráter filantrópico ou em serviços montados por associaçōes de familiares. $\mathrm{Na}$ rede pública de saúde, algumas crianças e adolescentes com autismo recebiam tratamento em "serviços-ilha", pouco integrados com os demais equipamentos de seu território, enquanto outras eram seguidas em ambulatórios tradicionais, com abordagem predominantemente medicamentosa (BRASIL, 2013; COUTO, 2004; MELLO et al., 2013). Muitas, entretanto, ficavam sem acompanhamento por quaisquer serviços, constituindo significativo contingente de desassistidos.

Entre os anos 1990 e 2000, algumas experiências locais, como a do CERSAMI em Betim-MG (FERREIRA; BONTEMPO, 2012), inspiraram a proposição dos Centros de Atenção Psicossociais Infanto-juvenis (CAPSi). Normatizados pelas Portarias no 336/2002 (BRASIL, 2002) e 3.088/2011 (BRASIL, 2013c), os CAPSi são serviços multidisciplinares de base territorial e forte articulação intersetorial, que oferecem atenção diária e intensiva prioritariamente a crianças e adolescentes portadores de transtornos mentais graves. Dentre essas psicopatologias, o autismo se destaca pelo seu início precoce e por estar associado a intenso prejuízo no estabelecimento de laços sociais, além da carga acarretada aos familiares ou outros responsáveis pelos cuidados cotidianos dessa população. Desta forma, os CAPSi podem ser considerados a primeira iniciativa a incluir 
o autismo, de modo destacado, embora não especializado, no campo da saúde mental pública do Brasil.

Ao lado dos CAPSi, há outros movimentos recentes mostrando que o autismo entrou definitivamente na pauta das políticas públicas no país. O principal deles é a sanção da Lei no 12.762/2012, instituindo a Política Nacional de Proteção dos Direitos da Pessoa com Transtorno do Espectro Autista (BRASIL, 2012). Mais recentemente, houve a publicação de dois documentos do Ministério da Saúde sobre o atendimento a pessoas com autismo no Sistema Único de Saúde (SUS), um deles coordenado pela área da Saúde Mental (BRASIL, 2013a) e o outro, pela área da Saúde da Pessoa com Deficiência (BRASIL, 2013b). Isso demonstra que as demandas de políticas para as pessoas com autismo se tornaram mais complexas, plurais e, por vezes, conflitantes, exigindo dos serviços, sejam CAPSI ou outros, o compromisso com a qualificação do cuidado oferecido aos usuários autistas e seus familiares.

No Brasil, ainda não foi realizado um esforço sistemático para avaliar o tratamento oferecido aos autistas nos serviços públicos de saúde mental, com vistas a se construir indicadores e analisadores da assistência prestada a esse grupo de usuários e suas famílias. Na verdade, são bastante recentes os trabalhos dedicados à prática avaliativa em serviços de saúde mental comunitários, no contexto da Reforma Psiquiátrica brasileira, destacando-se as pesquisas realizadas em CAPS III de Campinas-SP (ONOCKO-CAMPOS et al., 2008) e os projetos dedicados a avaliar CAPS na Região Sul do país (KANTORSKI et al., 2009; TOMASI et al., 2010). Tais pesquisas, contudo, não tomaram os CAPSi como objetos de estudos avaliativos, concentrando-se em CAPS I, II e III, dedicados à população adulta.

Couto (2012), em revisão metanarrativa da literatura sobre CAPSi publicada em periódicos brasileiros entre 2002 e 2010, encontrou 45 artigos que diziam respeito a esses serviços. Destes, apenas um foi classificado como "avaliativo", mas não tinha como objeto o tema do autismo. Em outros estudos, o autismo se fez presente quando o tema era a prevalência de diagnósticos nos CAPSi, ou quando discutiam o estatuto deste dispositivo como equipamento destinado preferencialmente a autistas (ao lado de psicoses e neuroses graves). A autora destaca que a significativa presença de crianças e adolescentes com autismo nos CAPSi contrasta com a ausência de informações sistematizadas sobre o trabalho ofertado nesses serviços. 
No cenário internacional, Brookman-Frazee, Taylor e Garland (2010) comentaram que, apesar de haver suporte empírico para diversos modelos específicos de intervenção, particularmente aqueles oferecidos em ambientes experimentais universitários, ainda se sabe pouco sobre sua aplicação em serviços de base comunitária. Payakachat et al. (2012), revisando instrumentos avaliativos disponíveis, apontaram a necessidade de estudos observacionais visando a construção e/ou aperfeiçoamento das medidas utilizadas para a avaliação da relação custo-efetividade de intervençôes terapêuticas e do impacto de diferentes sistemas de cuidado na evolução da criança com autismo. No contexto da pesquisa avaliativa qualitativa com profissionais, Brookman-Frazee et al. (2012) realizaram grupos focais com terapeutas de serviços comunitários de saúde mental de San Diego-EUA, os quais associaram sua frustração e os desafios no cuidado com a população autista a sua falta de treinamento sobre o tema. No registro da intersetorialidade, o Department of Education and Skills and Department of Health da Inglaterra (2002) publicou o documento Autistic Spectrum Disorder: good practice guidance, articulando estratégias entre as agências de educação e saúde, enquanto Brookman-Frazee et al. (2009) mostraram que os cuidados oferecidos a pessoas com autismo nos EUA estão dispersos por diversos setores, como educação, assistência social e justiça juvenil.

Nesse contexto, o presente trabalho, coordenado pelo Núcleo de Pesquisa em Políticas Públicas de Saúde Mental (NUPPSAM) do Instituto de Psiquiatria da Universidade Federal do Rio de Janeiro (IPUB/UFRJ), visou à criação de indicadores sobre o tratamento de autistas nos Centros de Atenção Psicossociais Infanto-juvenis (CAPSi), por meio de pesquisa avaliativa envolvendo profissionais de nível superior e médio e familiares de usuários autistas de 14 CAPSi da região metropolitana do Rio de Janeiro. ${ }^{1} \mathrm{O}$ caráter recente da implantação dos CAPSi e da tomada de responsabilidade no tratamento da clientela autista torna inadiável o estabelecimento de indicadores e analisadores que possam servir de guias e referências às boas práticas no cuidado com os autistas e suas famílias.

\section{Metodologia}

A metodologia da pesquisa avaliativa realizada em 14 CAPSi da região metropolitana do Rio de Janeiro foi adaptada daquela utilizada na pesquisa qualitativa, avaliativa e participativa realizada na rede de CAPS do município 
de Campinas-SP, descrita em Onocko-Campos et al. (2008). Tal metodologia implica a parceria entre pesquisadores e pessoas diretamente envolvidas com os serviços (em nosso caso, trabalhadores e familiares), a partir do reconhecimento do papel da participação na definição de políticas sociais, na articulação entre pesquisa e ação (FURTADO; ONOCKO-CAMPOS, 2008). Para atingir seus objetivos, a avaliação de programas sociais deve sempre trabalhar com um sistema de indicadores, sendo fundamental que estes surjam do processo de diálogo entre todos os sujeitos envolvidos (ASSIS et al., 2010; DEMO, 2002). A opção pela avaliação participativa costuma ser movida por razões pragmáticas - a expectativa de impacto direto na solução de problemas; políticas - a oportunidade de que indivíduos e grupos marginalizados sejam ouvidos na definição dos programas públicos; ou epistemológicas - a produção de conhecimento válido que leve em conta o contexto social (WEAVER; COUSINS, 2004). Tais razões não são excludentes, articulando efetividade, inclusão cidadã e construção compartilhada de saber, indo ao encontro dos princípios mais caros à Reforma Psiquiátrica brasileira.

Nesse referencial avaliativo, os grupos focais se destacam como método consagrado em pesquisas qualitativas em saúde e em ciências sociais, visando atingir um número suficiente de sujeitos representativos dos universos pesquisados, simultaneamente. Mais importante, o grupo focal permite, por meio da interação entre seus participantes, que a unidade de análise se desloque da opinião individual para o próprio grupo, o que possibilita acessar de modo mais apurado a visão da própria instituição e demais coletivos a respeito do tema pesquisado, com seus consensos, dissensos e contradições (KITZINGER, 2009; MIRANDA et al., 2008).

Antes do início do campo, houve o recrutamento de oito auxiliares de pesquisa, sendo que um deles funcionou como coordenador adjunto. Durante cerca de oito meses, foram realizadas reunióes semanais com a equipe da pesquisa, para que todos se familiarizassem com o tema principal e com a metodologia, elaborando o roteiro dos grupos focais. Em seguida, foi realizado contato telefônico com os coordenadores de saúde mental dos dez municípios e com os diretores dos 14 CAPSi envolvidos na pesquisa, visando sua apresentação e autorização para acesso às equipes dos serviços. Em seguida, foram visitados todos os CAPSi, durante sua reunião de equipe/supervisão, para a exposição dos objetivos e metodologia da pesquisa. A partir daí, foram agendados os 14 grupos focais que, por escolha 
dos próprios trabalhadores, foram todos marcados nos dias das reuniōes de equipe dos CAPSi, acontecendo paralelamente às mesmas. Os técnicos foram selecionados por meio de amostragem intencional, definida pela própria equipe e/ou a direção do serviço, obedecendo ao critério de estar há pelo menos seis meses trabalhando no CAPSi, não incluindo o próprio diretor/coordenador do serviço, num total de sete profissionais, obedecendo à seguinte composição em cada grupo: cinco técnicos de nível superior (1 médico, 1 enfermeira, 1 psicólogo e outros 2 escolhidos pelo CAPSi) e dois técnicos de nível médio. A inclusão de profissionais de diversas formações de nível superior e nível médio objetivou alcançar um equilíbrio entre homogeneidade e heterogeneidade na constituição de cada grupo (MIRANDA et al., 2008), permitindo que a variabilidade de posiçōes aparecesse, por detrás de possíveis posições consensuais. Isso levou à realização de 14 grupos focais com os técnicos de cada CAPSi, envolvendo, na prática, entre cinco a oito profissionais em cada um deles.

Os grupos focais com os familiares foram realizados por região, agrupando familiares dos CAPSi do município do Rio de Janeiro (Região Metropolitana I), dos municípios da Baixada Fluminense (Região Metropolitana I) e de Niterói, São Gonçalo e Itaboraí (Região Metropolitana II), totalizando três grupos focais. Os familiares também foram selecionados por amostragem intencional, sendo solicitado às equipes que escolhessem de dois a quatro familiares de crianças e adolescentes com autismo que estivessem em tratamento no CAPSi há pelo menos seis meses. Cada um desses grupos focais contou com a presença de sete a 12 participantes e ocorreu em local considerado de fácil acesso aos familiares, por transporte coletivo.

Cada grupo focal teve duração de uma hora e meia a duas horas e meia, contando com a presença de um moderador e um ou dois observadores, que tomavam notas mas também tinham a liberdade de fazer intervenções, comentários e perguntas esporádicas. A função de moderador nos 17 grupos foi dividida entre o coordenador e o coordenador adjunto, e a de observadores, entre os demais auxiliares de pesquisa. Os grupos focais se iniciavam com a apresentação dos objetivos da pesquisa, seguida da leitura e assinatura do TCLE, sendo que nos grupos com os familiares essa leitura aconteceu de modo coletivo, em voz alta. Partia-se então para a discussão, que era guiada pelo roteiro 
construído durante as reuniōes preparatórias da equipe da pesquisa. Tal roteiro não era seguido linearmente, respeitando-se a direção temática definida pelos participantes, mas em geral a maioria de seus tópicos era abordada. No caso dos familiares, foi necessária uma flexibilidade ainda maior por parte do moderador, pois com frequência os mesmos se mostravam mais interessados em fazer relatos pessoais sobre suas histórias e dificuldades nos cuidado com o filho autista, o que era respeitado e valorizado na condução do grupo, sem deixar com que o roteiro impedisse a livre manifestação.

A análise do material oriundo da transcrição dos registros em áudio dos grupos focais deu origem a cinco "temas ordenadores" (Organização do CAPSi; Projeto terapêutico individual; Atendimento e mobilização dos familiares; Rede, território e direitos; Formação dos profissionais e processos de trabalho). A síntese das discussões produzidas em torno dos temas ordenadores pelos 14 CAPSi e pelos três grupos de familiares foi então organizada em cinco "grades temáticas", cada uma reunindo os argumentos produzidos por cada grupo focal sobre um desses temas. A pesquisa foi então concluída com a realização de uma oficina final, durando um dia inteiro, reunindo a equipe organizadora e parte dos trabalhadores e familiares que participaram dos grupos focais. Nessa oficina, os participantes foram divididos em cinco subgrupos, cada um referente a um dos temas-ordenadores, sob a coordenação de um membro da equipe da pesquisa. Cada grupo trabalhou a partir da leitura do material sintetizado na "grade" de seu tema-ordenador, com a tarefa de construir indicadores que servissem de referência para o cuidado qualificado aos autistas e suas famílias, permitindo vislumbrar ações e monitorar seu andamento. Tais indicadores foram apresentados numa oficina final, sendo debatidos e/ou retificados pelos participantes. Os indicadores ganharam posteriormente uma redação final realizada pela equipe da pesquisa, visando atingir maior clareza e objetividade, sem alteração de seu conteúdo, garantindo que esses indicadores de fato representassem o produto final de um processo coletivo de trabalho, contemplando o ponto de vista de trabalhadores e familiares.

A pesquisa foi aprovada pelo Comitê de Ética em Pesquisa do IPUB/ UFRJ (parecer $n^{\circ}$ 81Liv3-11) e pelo Comitê de Ética em Pesquisa da Secretaria Municipal de Saúde e Defesa Civil do Rio de Janeiro (CAAE: 0045.0.249.314-11). Não houve exigência desse processo pelos demais municípios. 


\section{Resultados}

Em torno dos cinco temas ordenadores, foram produzidos cerca de 130 indicadores, todos eles apresentados sob a forma de perguntas. Deste modo, a resposta "sim" é aquela desejada, apontando a existência de cuidados de qualidade aos usuários com autismo e suas famílias e induzindo que estes sejam buscados quando ainda não estão presentes.

Iniciaremos cada eixo temático com uma descrição geral do contexto das discussōes e significados das questôes que deram origem aos indicadores.

\section{Tema: Organização do CAPSi}

Este tema implicou o debate sobre as questôes estruturais e os processos de trabalho nas unidades. Os profissionais tenderam a valorizar a flexibilidade na oferta de espaços de cuidado e a integração entre autistas e demais usuários. Já dentre os familiares, esta posição não formou um consenso, visto que parcela significativa demandou espaços exclusivos para os autistas, acreditando que necessitam de um trabalho específico e direcionado. Além disso, a maioria dos pais - e parte das equipes - considerou insuficiente a frequência e duração dos atendimentos, ressaltando também a precariedade estrutural de parcela dos CAPSi (quanto a sua conservação, tamanho e número das salas, localização, falta de telefone, carro e medicamentos, etc.). Os trabalhadores apontaram a necessidade do aumento no tamanho das equipes e os familiares se queixaram da alta rotatividade no quadro de funcionários e da ausência de algumas especialidades nos serviços. Tanto familiares quando as equipes ressaltaram as dificuldades na continuidade do tratamento e nos encaminhamentos dos autistas - especialmente para os CAPS - quando se tornam adultos.

\section{Subtema: Espaços individuais e coletivos de cuidado}

- As oficinas e demais espaços terapêuticos são construídos com a participação dos autistas, levando em conta seus interesses e habilidades?

- A equipe evita a padronização de atendimentos aos autistas no CAPSi?

- O serviço tem flexibilidade para conciliar a oferta de espaços coletivos com a de atendimentos individuais? 
- Há flexibilidade na revisão da frequência e na duração dos atendimentos de acordo com a demanda e necessidade do paciente (por exemplo, aumentando a frequência de idas ao CAPSi nas situações de crise)?

- Há possibilidade de atendimentos domiciliares para os pacientes que não conseguem frequentar o serviço?

- Há atividades externas no cuidado com os autistas?

- Na organização das festas, se toma medidas para facilitar a presença dos autistas e suas famílias?

\section{Subtema: Estrutura física/infraestrutura}

- Os espaços físicos dos serviços se adéquam às necessidades dos autistas e esse tema é discutido pela equipe em seus espaços de reunião?

- Há espaço físico destinado aos pais, dentro do serviço (sala de espera, sala para oficina de geração de renda, espaço para grupo de escuta ou troca de experiências, etc.)?

- O CAPSi tem autonomia gestora que lhe permita a compra de materiais diversificados de consumo, necessários no cotidiano do tratamento com os autistas e demais usuários (brinquedos, papéis, jardinagem, argila, culinária, etc.)?

- Há manutenção diferenciada para a estrutura física do CAPSi, devido à intensidade e particularidade de sua clientela, o que facilmente causa desgastes na casa na qual está o serviço?

- Há transporte exclusivo para uso do serviço?

- O espaço externo e interno do serviço é adequado para cadeirantes?

\section{Subtema: Integração e diversidade etária}

- Os espaços do CAPSi garantem a integração das crianças autistas com as não autistas?

- O CAPSi está preparado para receber a crescente demanda para tratamento de crianças autistas bem pequenas?

- A divisão dos espaços por faixa etária, caso existente, é flexível, respeitando as peculiaridades de cada caso e a avaliação do risco de conflitos entre crianças maiores e menores? 


\section{Subtema: "Porta de saida"}

- A equipe usa os espaços de reunião e supervisão para trabalhar suas dificuldades de encaminhar os pacientes autistas adultos para outros serviços?

- A equipe prepara os pais para o encaminhamento dos autistas adultos?

- O CAPSi tem um projeto articulado com o CAPS II ou III do território para a transferência dos autistas adultos?

- Os técnicos de referência assumem a tarefa da articulação da transferência dos autistas adultos, acompanhando-os ao CAPS ou ao outro serviço de destino?

- O CAPSi conta com um profissional de referência no CAPS que dê suporte no processo de passagem?

\section{Tema: Projeto Terapêutico Individual (PTI)}

As equipes destacaram a importância do técnico de referência, figura responsável pela elaboração do PTI, também realizando a ligação com outros técnicos e atendimentos aos pais. Contudo, a maioria dos profissionais sublinhou a importância de que o PTI da criança seja compartilhado por toda a equipe, não ficando restrito ao técnico de referência. No discurso das equipes, o PTI encontrou-se muito associado à frequência ao serviço e aos espaços internos e atividades externas nas quais a criança será inserida.

Os objetivos do tratamento foram entendidos de maneiras variadas pelos serviços, havendo ênfase nos efeitos positivos do CAPSi na socialização, interação interpessoal, criação de laços e autonomia dos autistas, mas também na redução da agressividade e das condutas repetitivas. Diversas equipes apontaram que o tratamento deve evitar ser invasivo, impositivo e diretivo, respeitando o tempo próprio dos autistas, sua subjetividade, o significado de seus comportamentos e valorizando os sutis sinais de melhora.

Quanto aos familiares, esses majoritariamente reconheceram as melhoras dos filhos, valorizando o CAPSi como espaço de convivência e socialização, mas também houve queixas de desconhecimento do "foco" do CAPSi e demandas por tratamento mais direcionado para o autismo. Os familiares reconheceram o esforço das equipes em desempenhar um bom trabalho apesar da precariedade estrutural de parte dos serviços. 
- O PTI é discutido e construído em equipe?

- Toda a equipe está ciente da direção do caso e do PTI de cada paciente autista?

- O território é levado em conta na elaboração do PTI?

- O PTI inclui necessariamente um trabalho intersetorial?

- Os PTIs são reavaliados ao longo do tratamento?

- O CAPSi inclui no PTI a avaliação dos diferentes fatores (financeiros, transporte, passe livre, etc.) que facilitam e dificultam o aumento da frequência da criança e da família ao serviço?

- O PTI é construído junto com os pacientes, visando à singularidade de cada caso, levando em consideração o tempo dos autistas, o que eles demandam e permitindo que eles mostrem como se deve tratá-los?

- O PTI é discutido e partilhado com os familiares?

- A reavaliação do PTI inclui as percepçõos vindas dos demais profissionais do CAPSi, além do técnico de referência?

\section{Subtema: Técnico de referência}

- Todo usuário autista tem um técnico de referência?

- O processo de trabalho no CAPSi permite que outros profissionais da equipe, além do técnico de referência, possam se autorizar a fazer intervenções clínicas com o paciente?

- A escolha do técnico de referência leva em conta as preferências, vínculos e afinidades demonstradas pela própria criança?

- O papel do técnico de referência inclui o atendimento dos familiares e o diálogo com outros setores?

- Trabalhadores de diversas categorias profissionais podem ocupar o papel de técnico de referência de um usuário autista?

\section{Subtema: Manejo das crises e internação}

- A crise é acolhida no CAPSi, fazendo do serviço o lugar do manejo das situações de urgência dos autistas?

- Os profissionais são capacitados para atender os momentos de crise da clientela autista? 
- Todos os profissionais atendem a situações de crise, e não apenas o médico?

- Há a possibilidade de a equipe do CAPSi fazer o acolhimento da crise fora do espaço do CAPSi?

- Há aumento da intensividade dos cuidados na crise?

- A equipe se esforça para contextualizar a crise e enxergá-la como expressão e trabalho psíquico da criança ou adolescente autista, ao invés de tomá-la como expressão inevitável da doença?

- As crises podem ser contornadas sem o uso de medicação, com a aplicação de outros recursos?

- A equipe esgota as abordagens para a crise antes de encaminhar a criança ou adolescente para a emergência psiquiátrica ou para a internação?

- Durante a internação, quando necessária, é feito um acompanhamento diário pela equipe do CAPSi no local em que ela é realizada?

\section{Subtema: Lugar da medicação}

- O tema da medicação circula entre os membros médicos e não médicos da equipe?

- A equipe avalia como importante que os profissionais não médicos entendam a função do medicamento?

- É explicada aos pais ou aos próprios usuários a função da medicação no tratamento?

- O PTI inclui a revisão periódica da medicação?

\section{Subtema: Objetivos do tratamento e percepção de seus efeitos}

- O tratamento tem promovido melhora na circulação em outros espaços além do circuito "casa-CAPSi-casa"?

- Na avaliação dos efeitos do tratamento, são incluídos como sinais de melhora: a ampliação da interação social do autista; a mudança da percepção das mães e pais sobre o filho; a maior adesão da família e entendimento sobre o tratamento; a melhora da auto e heteroagressividade; os avanços sutis da clínica do autismo?

\section{Tema: Atendimento e mobilização dos familiares}

Os profissionais dos CAPSi descreveram a oferta de atendimentos individuais e, principalmente, espaços grupais, em frequências e enquadres variados (na sala de 
espera, simultaneamente ou após o atendimento das crianças, etc.). Tais grupos, nos relatos das equipes, ora privilegiam a clínica e o manejo do sofrimento das mães e pais, ora enfatizam a divulgação de informações e esclarecimento de dúvidas, como aquelas ligadas às medicações. Os familiares reconheceram o acolhimento e amparo que recebem nos serviços, mas muitos se queixaram da falta de informações sobre a patologia dos filhos, levando-os a buscar conhecimento na internet e o dividir entre eles.

Poucos CAPSi citaram experiências de mobilização, organização ou participação dos familiares na gestão do serviço, com algumas referências a sua presença na organização de festas e outros eventos. Porém, nos grupos focais dos familiares, houve alguns relatos de engajamento político pontual e criação de associações, não restritas ao campo do autismo.

\section{Subtema: Estratégias individuais e grupais de cuidado}

- O cuidado com a família é incluído no PTI do paciente autista?

- A família participa da construção do PTI do paciente autista junto com a equipe?

- O PTI e o funcionamento do CAPSi são discutidos frequentemente com a família?

- Há grupos de família no CAPSi? Eles ocorrem várias vezes na semana, de modo a facilitar a presença dos pais?

- Há no CAPSi atendimento individual para os pais?

- Os atendimentos com familiares, caso existam, têm repercutido na melhora do quadro das crianças?

- As famílias reconhecem seu técnico de referência como uma referência de fato, acessível a elas no cotidiano do serviço, e não apenas como designação burocrática?

- Quando a equipe do CAPSi avalia ser necessário um atendimento individual para os responsáveis em outro serviço, é feito um encaminhamento de forma implicada?

- Existe um espaço físico para os familiares serem acolhidos?

- Há alguma atividade regular de acolhimento aos pais na sala de espera/recepção?

- O CAPSi consegue elaborar estratégias de atendimento aos pais quando há resistência por parte deles ao tratamento? 
- Faz parte do PTI a construção, junto aos pais e demais responsáveis, de outras possibilidades e interesses de vida que não se limitem aos cuidados do filho?

- A questão da sobrecarga dos familiares é considerada na elaboração do PTI e no manejo cotidiano do caso, havendo estratégias para aliviá-la?

- O CAPSi estimula a criação de espaços de convivência e sociabilidade para os pais, dentro e fora do serviço?

- É discutida a questão do benefício de prestação continuada (BPC) com os pais, levando em consideração o PTI de cada paciente?

- Em um eventual processo de internação do usuário autista, os pais são acompanhados pela equipe do CAPSi?

\section{Subtema: Espaços para informação e educação em saúde}

- O CAPSi inclui a família nas estratégias de articulação com a rede psicossocial ampliada (escola, CRAS, conselho tutelar, etc.)?

- O CAPSi desenvolve espaços de discussão com os pais sobre temáticas relativas à saúde, como sexualidade, higiene, medicação, diagnóstico, etc.?

- O CAPSi esclarece os familiares sobre os direitos e benefícios sociais do paciente autista?

- O CAPSi desenvolve espaços de discussão com os pais sobre direitos do paciente?

\section{Subtema: Iniciativas de cidadania, mobilização e organização}

- Existe no CAPSi alguma estratégia de geração de renda para os pais?

- O CAPSi desenvolve assembleias de familiares?

- O CAPSi estimula os familiares a exercerem uma participação na política pública?

- O CAPSi estimula os familiares a se integrarem formando grupos, associações ou cooperativas?

\section{Subtema: Participação na gestão do CAPSi}

- Os familiares participam das decisões sobre a organização e gestão do serviço?

- Os pais são convocados a ajudar a cuidar do espaço do CAPSi? São escutados no momento da realização de obras no serviço?

- Os familiares participam da organização de atividades recreativas e comemorativas do serviço (festas, etc.)? 


\section{Tema: Rede, território e direitos}

Vários familiares contaram sobre seu histórico de peregrinação por diversos serviços, mas a maior parte deles reconheceu a rapidez em conseguir atendimento, após acessarem o CAPSi. As equipes apontaram como principais problemas de acesso a imensidão dos territórios de abrangência dos serviços e a malha inadequada de transportes coletivos, enquanto os familiares criticaram, além desses fatores, os horários de atendimento que coincidiam com seus turnos de trabalho. A articulação com a educação se destacou no tópico da intersetorialidade, mas os contatos com as escolas se davam mais a partir dos casos do que em torno de parcerias institucionalizadas.

Os profissionais apresentaram diferentes posturas sobre os tratamentos (como neurologia, fonoaudiologia e equoterapia) feitos concomitantemente àquele provido pelos CAPSi, avaliando que algumas vezes os trabalhos se completavam, mas em outras se tornavam concorrentes, havendo dificuldades na comunicação entre diferentes serviços. Equipes e familiares relataram dificuldades para conseguir atendimentos na rede pública de saúde (pediatria, odontologia, exames laboratoriais), muitas vezes ligadas ao estigma da "agitação" dos autistas.

Na discussão sobre o "Benefício de Prestação Continuada" (BPC), as equipes reconheceram a ajuda na melhoria dos quadros de pobreza das famílias, mas também o associaram a entraves no tratamento de algumas crianças, que acabavam marcadas pelo estigma da incapacidade ou abandonavam o serviço, e muitos profissionais consideraram importante avaliar essa questão no "caso a caso". Alguns familiares relataram dificuldades em conseguir o laudo para o $\mathrm{BPC}$ ou mesmo informações sobre ele nos CAPSi, levando os pais e mães a orientarem uns aos outros sobre as formas de obtê-lo.

\section{Subtema: Cobertura e acesso ao serviço}

- O CAPSi é bem localizado no município, em região de fácil acesso?

- O CAPSi conta com boa cobertura da rede de transportes públicos?

- O CAPSi conta com transporte próprio para deslocamentos de equipe e usuários?

- O CAPSi de seu município/região está designado a uma cobertura populacional de acordo com a Portaria Ministerial no 3.088/2011 ou outras norma- 
tizações vigentes? Ele é suficiente para a cobertura adequada da demanda do território a ele atribuído?

- O CAPSi é conhecido pela população? Ele promove a divulgação de seus serviços para a população?

- O CAPSi tem estratégias para ser reconhecido pela comunidade em seu entorno e pelos demais equipamentos públicos do território como o lugar adequado para o tratamento dos autistas?

- O CAPSi se concebe como lugar primordial de acolhimento para os autistas sem ter que, necessariamente, assumir o tratamento de todos os autistas do território que chegam ao serviço?

- O CAPSi dá conta das especificidades e particularidades da clínica com os autistas sem se transformar em serviço ambulatorial ultraespecializado e não territorial?

\section{Subtema: Articulação com a rede pública e a comunidade}

- Os demais equipamentos da rede (escolas, justiça, assistência social, abrigos, conselhos tutelares, etc.) conhecem o trabalho do CAPSi? Esses equipamentos reconhecem o CAPSi como local de referência no atendimento da clientela autista?

- O CAPSi contribui para a educação da população e de profissionais da rede ampliada (professores, conselheiros tutelares, outros profissionais de saúde, etc.) quanto ao autismo?

- A equipe do CAPSi consegue conjugar o cuidado oferecido dentro do CAPSi com a articulação intersetorial?

- As parcerias com a rede conseguem avançar a partir dos contatos pontuais e informais (dependendo de iniciativas de cada profissional e das necessidades de cada caso), para se estabelecerem de maneira sólida, regular e formal (com consolidação das parcerias institucionais)?

- O CAPSi mantém diálogo, reuniões e intercâmbio com os demais equipamentos da rede, de modo regular (escolas, justiça, assistência social, abrigos, conselhos tutelares, etc.)?

- O CAPSi mantém um diálogo, reuniōes e intercâmbio com as equipes do Programa de Saúde da Família, de modo regular? Há cuidado compartilhado nos casos cobertos pelo PSF? 
- O CAPSi auxilia os autistas e seus familiares no acesso aos demais dispositivos de saúde (hospitais clínicos, dentistas, exames laboratoriais, etc.)?

- A articulação do CAPSi com os possíveis atendimentos complementares feitos na rede (como neurologistas, fonoaudiólogos, equoterapia, por exemplo) ocorre de maneira satisfatória, com diálogo e troca de informações?

- O território do CAPSi conta com esses serviços complementares, evitando um grande deslocamento da família para consegui-los?

- O CAPSi participa regularmente de algum fórum intersetorial? Consegue utilizar esse espaço para a consolidação de parcerias?

- O CAPSi considera as parcerias com os outros equipamentos eficientes?

- O CAPSi utiliza os recursos comunitários, esportivos e culturais (casas de cultura, vilas olímpicas, etc.) quando estabelece o PTI dos autistas?

- O CAPSi promove eventos com o objetivo de integrar a comunidade ao serviço e seus usuários? Eles ocorrem com frequência regular?

\section{Subtema: Direitos e benefícios sociais}

- A discussão sobre concessão dos benefícios - Benefício de Prestação Continuada (BPC), Passe Livre ou outros - está incluída em cada PTI? Há discussão sobre o momento e as condições para essa concessão, em cada caso?

- O CAPSi auxilia os usuários e familiares na obtenção desses benefícios, quando esses são indicados, junto aos órgãos responsáveis?

- Há espaços para avaliar se a concessão do BPC favoreceu o tratamento, ou seja, se houve avanço no tratamento após sua concessão?

- O laudo fornecido para o Passe Livre contempla todas as necessidades de transporte para o tratamento e também para o acesso a atividades culturais, esportivas, educacionais, etc.?

\section{Tema: Formação dos profissionais e processos de trabalho}

A defesa da importância de uma "linha teórica mínima" conviveu, no discurso dos profissionais, com elogios à diversidade de linhas, com a crítica ao enrijecimento teórico e com posiçôes de técnicos que afirmavam pautar sua prática mais em suas próprias experiências e insights. A psicanálise foi o referencial teórico mais citado, sendo também mencionadas outras correntes (como Gestalt e TCC). 
Houve concepções de autismo muito variadas, mas também muitas críticas a noções deficitárias ou da visão do autista como ser isolado.

Os CAPSi reconheceram a importância de grupos de estudo, especializações e cursos, embora vários profissionais apontassem que "não há saber posto" e que o trabalho no serviço já tinha um papel formador, com destaque para a supervisão clínico-institucional. As equipes foram unânimes ao valorizar a integração e circulação dos saberes de cada especialidade no coletivo de trabalhadores, embora percebessem dificuldades dos familiares em entender o papel de cada profissional. Os médicos e enfermeiros foram citados como aqueles que acabavam preservando uma identidade e práticas próprias, por vezes excessivamente destacadas da equipe.

\section{Subtema: Capacitação, estudos e formação:}

- O CAPSi possui um espaço para grupo de estudos que aborde temas articulados com a prática diária? Esse espaço também é usado para reflexões sobre o papel político do CAPSi na sociedade?

- O CAPSi funciona como um lugar de construção e produção de um saber teórico-clínico? Esse saber é partilhado com outros serviços e espaços de discussão?

- Os profissionais do serviço têm conhecimento da legislação da Reforma Psiquiátrica e dos princípios e diretrizes do SUS?

- A prática clínica realizada no CAPSi está em consonância com a política pública nacional de Saúde Mental de Crianças e Adolescentes?

- Os profissionais que atuam no CAPSi têm formação no campo da atenção psicossocial?

- Os profissionais do CAPSi frequentam outros espaços de discussão, sejam políticos, clínicos ou acadêmicos?

- Há apoio do CAPSi para a busca de formação/capacitação da equipe?

- Há pessoas que fazem capacitação subsidiada pelo serviço ou pela gestão local?

- Os trabalhadores que realizaram algum tipo de capacitação trazem um retorno para equipe sobre seu aprendizado?

- O CAPSi busca divulgar sua produção teórico-clínica em meios científicos?

\section{Subtema: Circulação do conhecimento e processo de trabalho}

- O CAPSi tem uma direção clínica que permite a circulação do saber entre os membros da equipe?

- A construção do diagnóstico é realizada em equipe? 
- O CAPSi investe na promoção de relações horizontais entre os diversos técnicos, de modo a evitar que algum profissional ocupe um lugar hierarquicamente superior em relação aos demais?

- A direção clínica coletiva da equipe prevalece sobre as estratégias de cada especialidade profissional, de modo a evitar a fragmentação do PTI?

- As atuações dos profissionais vão além do campo tradicional de suas respectivas especialidades?

- Os profissionais conseguem articular as questôes psicossociais com aquelas específicas de sua formação acadêmica?

- O cuidado com os autistas é compartilhado pela equipe, independentemente da especificidade da formação do profissional?

- O médico se inclui na equipe como técnico de saúde mental, ou seja, não limitando suas intervençỗes aos campos do diagnóstico e medicação?

- A organização do serviço possibilita que todos os profissionais, das diversas categorias presentes no CAPSi, participem da recepção ou porta de entrada?

- Todos os profissionais, inclusive o médico, participam dos dispositivos coletivos oferecidos pelo serviço?

- O CAPSi tem a equipe mínima de funcionários prevista na Portaria GM no 336/2002 e demais normatizaçóes vigentes, incluindo pelo menos um médico permanentemente?

- O aumento no número de profissionais no serviço, quando ocorre, leva também ao aumento nos projetos externos do CAPSi?

- Há critérios claros para avaliar a entrada do profissional no CAPSi?

- Há projetos para evitar a rotatividade dos profissionais no CAPSi, caso isso esteja afetando a qualidade do atendimento dos autistas?

\section{Subtema: Supervisão clínico-institucional}

- Há supervisão clínico-institucional no CAPSi? É um espaço formador e de construção coletiva de trabalho?

- Todos os profissionais participam da supervisão/reunião de equipe?

- A supervisão clínico-institucional tem produzido mudanças no trabalho da equipe com os autistas?

- A angústia dos profissionais em relação às dificuldades na clínica com os autistas é trabalhada em supervisão? 


\section{Subtema: Concepções de autismo}

- A equipe do CAPSi debate sobre os referenciais teóricos presentes de forma explícita ou implícita no cuidado oferecido pelo serviço?

- A equipe do CAPSi percebe de que maneiras as concepções de autismo presentes no serviço afetam as famílias dos usuários e o território?

- Há abertura no CAPSi para concepções que tomam o autismo como uma posição subjetiva que pode implicar sofrimento psíquico?

\section{Discussão}

Os indicadores apresentados acima resultam de uma produção coletiva que envolveu trabalhadores de 14 CAPSi e familiares de crianças e adolescentes com autismo neles atendidos. Seu principal valor é terem emergido do próprio campo da atenção psicossocial infanto-juvenil do SUS, ao invés de serem propostos ou impostos por instâncias de fora. Durante o percurso da pesquisa, a metodologia participativa garantiu, por meio dos grupos focais e da oficina final, que o resultado final de fato representasse de modo fidedigno a voz dos participantes. Vale destacar que, a despeito de certas discordâncias entre as posiçôes de profissionais e familiares, reveladas no decorrer dos grupos focais, as concordâncias permitiram a elaboração consensual dos indicadores, durante a oficina final.

No processo de elaboração final do resultado da pesquisa, realizado pela equipe organizadora, alguns indicadores foram realocados de seu eixo temático original, outros tiveram sua redação levemente alterada, alguns foram amalgamados e outros foram desmembrados, mas não houve nenhum indicador acrescentado posteriormente. Muitos se desdobravam em uma segunda pergunta na formulação original, e isso foi mantido na redação final de alguns deles. É possível verificar que certas questóes se repetem em diferentes eixos temáticos, o que pode ser considerado sinal de sua transversalidade no cotidiano do serviço e de sua relevância na tarefa avaliativa. A divisão em temas e subtemas serve para facilitar sua aplicação no cotidiano dos serviços e nos parece representativa da variedade de tópicos implicados no cuidado de usuários com autismo.

Mesmo que o escopo da pesquisa inclua municípios e serviços heterogêneos, o recorte escolhido faz com que os indicadores tenham forte caráter local, pois foram construídos a partir de CAPSi localizados em municípios geograficamente contíguos, com histórico e inserção próprios no campo da saúde mental, da 
Reforma Psiquiátrica e do próprio SUS. Apesar disso, apostamos em seu poder de generalização para outros CAPSi do Brasil, o que apenas sua aplicação em ações e pesquisas futuras poderá constatar ou refutar. De todo modo, a existência de tais indicadores, num cenário no qual eles ainda não operavam, é a principal contribuição da pesquisa ao campo da atenção psicossocial, e acreditamos que eles podem ser aplicados na avaliação do trabalho dos CAPSi com um todo, pois muitos não são específicos para as crianças e adolescentes com autismo. Em acréscimo, oferecem um retrato das concepções e ideais dos trabalhadores e familiares a respeito do tratamento oferecido nos CAPSi, servindo como analisadores do atual modo de organização dos serviços e das modificações necessárias.

Como comentam Onocko et al. (2008) a respeito do resultado da pesquisa realizada nos CAPS de Campinas, os indicadores podem servir como "roteiro de boas práticas” para os CAPSi, desde que não se deduza daí um caráter prescritivo ou normativo, no sentido forte desses termos. Da mesma forma, não é esperada sua transformação em instrumental quantitativo, fazendo com que certo número de respostas "sim" deem origem a "patamares de qualidade", gerando a busca e a competição irrefletidas entre os CAPSi para alcançá-los ou a hierarquização entre esses serviços. Espera-se, isso sim, que os indicadores sejam apropriados como instrumental analítico pelos próprios trabalhadores, familiares e diretores dos CAPSi para auxiliar no planejamento de açôes e no monitoramento de seu andamento, podendo ter a mesma utilidade para os gestores municipais, estaduais e federais. Sua existência serve como indutora de avanços na oferta de atenção psicossocial às crianças e adolescentes com autismo nos CAPSi.

A pesquisa avaliativa dirigida aos novos serviços de saúde mental, especialmente aqueles destinados a crianças e adolescentes, ainda tem muito a avançar no Brasil. As lacunas relacionadas ao tópico da avaliação qualitativa de serviços de base comunitária e territorial para pessoas com autismo ficam patentes, dentro e fora do país. Muitos trabalhos da literatura internacional se dirigem a avaliar a efetividade de abordagens terapêuticas ou psicossociais específicas, nem sempre em serviços públicos comunitários e em sistemas de saúde diferentes do brasileiro, com destaque para o norte-americano, tornando difícil a comparação entre as diferentes realidades nacionais.

A existência de pesquisas como a nossa e a disseminação de seus resultados devem também servir para induzir futuras pesquisas na parceria entre a 
universidade e o SUS, na tarefa permanente de produção de conhecimento e formação para a Reforma Psiquiátrica. No caso do autismo, a qualificação do cuidado e a pesquisa de sua efetividade, nos CAPSi e demais pontos da Rede de Atenção Psicossocial, deve se transformar em objetivo comum dos formuladores das políticas públicas, trabalhadores, familiares e usuários dos serviços. ${ }^{2}$

\section{Agradecimentos}

Agradecemos aos auxiliares de pesquisa Maria Luiza Iusten da Silva, Rafael Perricone Fischer, Andreia da Silva Miguel, Marília de Albuquerque Torres, Vanessa Kaiel, Aline Rodrigues Alves Fernandes e Juliana César Machado, pela valiosa contribuição em todo o processo da pesquisa, especialmente nos grupos focais, na transcrição das gravações e na oficina final.

\section{Referências}

ASSIS, S.G. et al. Definição de objetivos e construção de indicadores visando à triangulação. In: MINAYO, M.C.S.; ASSIS, S.G.; SOUZA, R.E. (Org.). Avaliação por triangulação de métodos: abordagens de programas sociais. Rio de Janeiro: Fiocruz, 2005.

BLAXILL, M.F. What's going on? The question of time trends in autism. Public Health Reports, v. 119, p. 536-551, 2004.

BRASIL. Lei no 12.764, de 27 de dezembro de 2012. Institui a Política Nacional de Proteção dos Direitos da Pessoa com Transtorno do Espectro Autista. Diário Oficial [da] República Federativa do Brasil, Brasília, DF, 2012.

. Ministério da Saúde. Diretrizes de Atenção à Reabilitação da Pessoa com Transtornos do Espectro do Autismo. Brasília: Ministério da Saúde, 2013b.

Ministério da Saúde. Linha de cuidado para a atenção às pessoas com Transtornos do Espectro do Autismo e suas famílias na Rede de Atenção Psicossocial do SUS. Brasília: Ministério da Saúde, 2013a.

Portaria GM no 3.088, de 23 de dezembro de 2011 (republicada em 21 de maio de 2013). Institui a Rede de Atenção Psicossocial para pessoas com sofrimento ou transtorno mental, incluindo aquelas com necessidades decorrentes do uso de crack, álcool e outras drogas, no âmbito do Sistema Único de Saúde (SUS). Diário Oficial [da] República Federativa do Brasil, Brasília, DF, 2013c.

Portaria GM no 336, de 19 de fevereiro de 2002. Normatiza os CAPS I, CAPS

II, CAPS III, CAPS i II e CAPS ad II. Diário Oficial [da] República Federativa do Brasil, Brasília, DF, 2002. 
BROOKMAN-FRAZEE, L. et al. Involvement of youths with autism spectrum disorders or intellectual disabilities in multiple public service systems. Mental Health Research in Intellectual Disabilities, v. 2, p. 201-219, 2009.

. Therapist perspectives on community mental health services for children with autism spectrum disorders. Adm Policy Ment Health, v. 39, p. 365-373, 2012.

BROOKMAN-FRAZEE, L.; TAYLOR, R.; GARLAND, A.F. Characterizing communitybased mental health services for children with autism spectrum disorders and disruptive behavior problems. J Autism Dev Disord, v. 40. p. 1.188-1.201, 2010.

BRUGHA, T.S. et al. Epidemiology of autism spectrum disorders in adults in the community in England. Archives of General Psychiatry, Chicago, v. 68, n. 5, p. 469-465, 2011.

CENTERS FOR DISEASE CONTROL AND PREVENTION. Prevalence of autism spectrum disorders: autism and developmental disabilities monitoring network, 14 sites, United States, 2008. Morbidity and Mortality Weekly Report: surveillance summaries, v. 61, n. 3, mar. 2012.

CHARMAN, T. The prevalence of autism spectrum disorders: recent evidence and future challenges. European Child \& Adolescent Psychiatry, v. 11, n. 6, p. 249-256, 2002.

COO, H. et al. Trends in autism prevalence: diagnostic substitution revisited. J Autism Dev Disord, v. 38, n. 6, p. 1036-1046, 2008.

COUTO, M.C.V. Política de Saúde Mental para crianças e adolescentes: especificidades e desafios da experiência brasileira (2001-2010). 2012. Tese (Doutorado em Saúde Mental) - Instituto de Psiquiatria da Universidade Federal do Rio de Janeiro, Rio de Janeiro, 2012.

Por uma política pública de saúde mental para crianças e adolescentes. In: FERREIRA, T. (Org.). A criança e a saúde mental: enlaces entre a clínica e a política. Belo Horizonte: Autêntica, 2004. p. 61-72.

DEMO, P. Avaliação qualitativa. São Paulo: Cortez, 2002.

DEPARTMENT FOR EDUCATION AND SKILLS, DEPATMENT OF HEALTH. Autistic Spectrum Disorders good practice guidance. Nottingham: DfES Publications, 2012

FERREIRA, T.; BONTEMPO, V.L. (Org.) Crianças e adolescentes: o cuidado em saúde mental, o trabalho feito por muitos. Belo Horizonte: CRV, 2012.

FOMBONNE, E. The prevalence of autism. The Journal of the American Medical Association, n. 289, p. 87-89, 2003.

FURTADO, J.P.; ONOCKO-CAMPOS, R. Participação, produção de conhecimento e pesquisa avaliativa: a inserção de diferentes atores em uma investigação em saúde mental. Cad. Saúde Pública, Rio de Janeiro, v. 24, n. 11, p. 2.671-2.680, 2008.

KANTORSKI, L.P. et al. Contribuições do estudo de avaliação dos centros de atenção psicossocial da região Sul do Brasil. Cad. Bras. Saúde Mental, v. 1, n.1, 2009 (CD-Rom). 
KITZINGER, J. Grupos focais. In: POPE, C.; MAYS, N. Pesquisa qualitativa na atenção à saúde. Porto Alegre: Artmed, 2009. p. 33-43.

MELLO, A.M. et al. Retratos do autismo no Brasil. São Paulo: Associação dos Amigos do Autista, 2013.

MIRANDA, L. et al. Dos grupos focais aos grupos focais narrativos: a descoberta no caminho da pesquisa. In: ONOCKO-CAMPOS, R. et al. (Org.). Pesquisa avaliativa em saúde mental: desenho participativo e efeitos da narratividade. São Paulo: Aderaldo \& Rothschhild, 2008. p. 249-277.

ONOCKO-CAMPOS, R. et al. Pesquisa avaliativa em saúde mental: desenho participativo e efeitos da narratividade. São Paulo: Aderaldo \& Rothschhild, 2008.

PAULA, C.S. et al. Brief report: prevalence of pervasive developmental disorder in Brazil: a pilot study. Journal of Autism and Developmental Disorders, New York, v. 41, n. 12, p.17381742, 2011.

PAYAKACHAT, N. et al. Autism spectrum disorders: a review of measures for clinical, health services and cost-effectiveness applications. Expert Rev Pharmacoecon Outcomes Res., v. 12, n. 4, p. 48-503, 2012.

TOMASI, E. et al. Efetividade dos Centros de Atenção Psicossocial no cuidado a portadores de sofrimento psíquico em cidade de porte médio do Sul do Brasil: uma análise estratificada. Cad. Saúde Pública, Rio de Janeiro, v. 26, n. 4, p. 807-815, 2010.

WEAVER, L.; COUSINS, B. Unpacking the participatory process. Journal of Multidisciplinary evaluation, v. 1, p. 19-40, 2004.

\section{Notas}

${ }^{1}$ Os serviços participantes, que representavam todos os CAPSi em funcionamento na região metropolitana do Rio de Janeiro no início da pesquisa, foram: CAPSi Pequeno Hans, CAPSi Eliza Santa Roza, CAPSi Maria Clara Machado, CAPSi João de Barro e CAPSi CARIM (todos na cidade do Rio de Janeiro); CAPSij DC (Duque de Caxias); CAPSi D. Adriano Hipólito (Nova Iguaçu); CAPSi Dr. João Dansa Ribeiro (São João de Meriti); CAPSi Casinha Azul (Itaguaí), CAPSi Queimados (Queimados); CAPSi Belford Roxo (Belford Roxo); CAPSi Monteiro Lobato (Niterói); CAPSi João Caetano (Itaboraí); e CAPSi Zé Garoto (São Gonçalo).

${ }^{2}$ R.C. Lima participou da concepção da pesquisa; coleta e análise dos dados; elaboração e finalização do artigo. M.C.V. Couto e P.G.G. Delgado participaram da concepção da pesquisa; da revisão do artigo; e atuaram como consultores. B.D.C de Oliveira participou da concepção da pesquisa; coleta e análise dos dados; redação do artigo. 


\section{Abstract}

Indicators on the mental health care of autistic children and adolescents in the CAPSi of Rio de Janeiro's metropolitan region

The Centers of Psychosocial Care for Children and Adolescents (CAPSi) are strategic services for treatment of children and adolescents with mental problems, including autism spectrum disorders. This research aimed at creating indicators on the treatment of autistic children and adolescents in CAPSi. The participative research involved mental health workers and parents of children from 14 CAPSi in Rio de Janeiro's metropolitan region. The methodology involved 14 focal groups with workers and three focal groups with parents of patients. The transcriptions of audio recordings of focal groups engendered five "organizers themes", that were elaborated in a final workshop, generating about 130 indicators, which were grouped around five axis: service organization; individual therapeutic project; territorial network and advocacy; family support and organization; professional training and work process. These indicators can serve as guides and references to best practices in caring for people with autism and their families, and can be used in future evaluations of the treatment offered in the CAPSi.

Key words: evaluative research; autism; Centers of Psychosocial Care; child and adolescent mental health. 